Paediatr Paedolog 2016 51:104-108

DOI 10.1007/s00608-016-0371-y

Online publiziert: 10. Juni 2016

(c) The Author(s) 2016. This article is available

at SpringerLink with Open Access

CrossMark

\author{
Christina Pöppelmeyer ${ }^{1}$ Oliver Helk ${ }^{1}$ Sarah Mehany' $\cdot$ Richard Hauer ${ }^{2}$. \\ Otmar Pachinger ${ }^{3} \cdot$ Kurt Widhalm $^{1}$ \\ 'Österreichisches Akademisches Institut für Ernährungsmedizin, Wien, Österreich \\ ${ }^{2}$ Institut für Sportwissenschaften der Universität Wien, Wien, Österreich \\ ${ }^{3}$ Österreichischer Herzfonds, Wien, Österreich
}

\title{
Die Wiener Präventionsstudie EDDY - Erste Ergebnisse
}

Die teilnehmenden Schüler verteilen sich auf zwei Wiener Gymnasien und zwei Wiener Mittelschulen, deren Klassen randomisiert der Interventions- oder Kontrollgruppe zugeordnet wurden. Insgesamt partizipierten $n=82$ Schüler an der Intervention, $n=59$ Schüler waren der Kontrollgruppe zugeordnet (•Tab. 1).

\section{Intervention}

Die Interventionsgruppe erhielt über zwei Semester eine altersgerechte Ernährungsschulung im Ausmaß von 20 Stunden sowie eine medizinische Schulung (fünf Stunden) und eine 20-stündige Sport- und Bewegungsintervention.

Im Rahmen der medizinischen Schulung erwarben die Probanden Grundlagenwissen $\mathrm{zu}$ physiologischen und biochemischen Funktionsabläufen im menschlichen Körper. Dabei wurden die Funktionsweisen und Aufgaben des Skelettapparates, der Muskulatur, des Fettgewebes und der Hormone sowie die Regulation des Blutzuckerspiegels besprochen.

Die Schulungen zum Thema "gesunde Ernährung" gliederten sich in zwei Zyklen. In einem ersten Schulungszyklus wurden grundlegende Begriffe der Ernährungslehre überwiegend theoretisch erarbeitet. $\mathrm{Zu}$ den Lehrinhalten dieses Themenblocks zählten die Wirkung der Makro- und Mikronährstoffe, die Bedeutung einer regelmäßigen Nahrungs- und Flüssigkeitszufuhr für die tägliche Leistungsfähigkeit sowie der Ursprung von Lebensmitteln. Darüber hinaus wurden auch der Aufbau der
Ernährungspyramide, die Kennzeichnung von Lebensmittelverpackungen und die Wahl von „guten“ und „schlechten“ Durstlöschern besprochen. In einem zweiten Schulungszyklus wurde das erworbene Wissen durch praktische Erfahrungen vertieft. Die Schüler führten Experimente durch, in denen sie verschiedene Lebensmittel mit verbundenen Augen „erschmeckten“, den Wasser- und Fettgehalt von unterschiedlichen Nahrungsmitteln visualisierten, verarbeiteten Lebensmitteln ihre ursprünglichen Rohstoffe zuordneten und gemeinsam gesunde Snacks und Mahlzeiten zubereiteten.

Die Sport- und Bewegungsintervention diente vor allem dazu, die Schüler durch Bewegungs- und Koordinationsspiele spielerisch zur Integration von Bewegung in den täglichen Lebensalltag zu animieren.

\section{Testungen}

Vor und nach Intervention sowie bei Follow-up-Messungen nach sechs und zwölf Monaten wurden die Probanden körperlich vermessen und die psychische Konstitution, das Ernährungswissen und -verhalten sowie die körperliche Fitness erhoben. Zudem wurde den Probanden zur Bestimmung des Gesundheitszustandes Blut abgenommen.

\section{Körperkomposition}

Mittels Stadiometer wurde die Körpergröße der Probanden gemessen. Zur Klassifizierung der Körperkomposition in die BMI-Perzentile für Kinder
Die Studienpopulation umfasste $n=141$ Probanden im Alter von 11-14 Jahren. 


\begin{tabular}{|llll|}
\hline Tab. 1 & Aufteilung der Studienpopulation & & \\
\hline & $\boldsymbol{n}$ & Männlich & Weiblich \\
\hline Interventionsgruppe & 82 & 41 & 41 \\
\hline Kontrollgruppe & 59 & 34 & 25 \\
\hline
\end{tabular}

\begin{tabular}{|c|c|c|c|}
\hline & $n$ & $\begin{array}{l}\text { Häufigkeit pathologischer } \\
\text { Werte vor Intervention in \% }\end{array}$ & Normwerte \\
\hline Triglyceride & 139 & 29,50 & $<90 \mathrm{mg} / \mathrm{dl}^{1}$ \\
\hline LDL-Cholesterin & 139 & 13,67 & $<100 \mathrm{mg} / \mathrm{dl}^{1}$ \\
\hline Lipoprotein (a) & 139 & 24,46 & $<30 \mathrm{mg} / \mathrm{dl}^{1}$ \\
\hline Vitamin $B_{12}$ & 139 & 17,98 & $263-1170 \mathrm{pg} / \mathrm{ml}^{2}$ \\
\hline Vitamin D & 138 & 86,95 & $\geq 75 \mathrm{nmol} / \mathrm{I}^{3}$ \\
\hline Ferritin & 139 & 42,45 & $\geq 15 \mu \mathrm{g} / \mathrm{I}^{1}$ \\
\hline $\begin{array}{l}{ }^{1} \text { Normwert aus [11] } \\
{ }^{2} \text { Normwerte aus [12] } \\
{ }^{3} \text { Normwert aus [13] }\end{array}$ & & & \\
\hline
\end{tabular}

und Jugendliche nach Kromeyer-Hauschild [5] wurden das Körpergewicht, der Körperfettanteil sowie der Muskelund Wasseranteil des Körpers mittels bioelektrischer Impendanzanalyse ermittelt.

\section{Blutparameter}

Anhand der Parameter Cholesterin, Triglyceride, LDL-Cholesterin, HDL-Cholesterin, hs-Insulin, Eisen, Vitamin A, D, E, K, C, B $1,6,12$, Folsäure, Leukozyten, Lymphozyten, Eosinophile, Selen und $\beta$-Carotin wurde der Gesundheitsstatus der Probanden bestimmt.

\section{Ernährungswissen}

Das Ernährungswissen wurde mittels eines eigens konzipierten Quiz „Was weißt $d u$ über Ernährung", basierend auf zwölf altersgerechten Fragen über Nahrungsmittel und Nahrungsmittelinhaltsstoffe, erhoben.

\section{Ernährungsverhalten}

Ernährungsverhalten und Ernährungspräferenzen wurden anhand eines standardisierten Fragebogens (Food Frequency Questionnaire für Kinder und Jugendliche) retrospektiv ermittelt.

\section{Psychische Konstitution}

Psychologische sowie sportwissenschaftliche und ernährungsassoziierte Einflussfaktoren auf die Entstehung von Übergewicht und Adipositas wurden anhand des interdisziplinären Testsystems „ADEVA“ erfasst [5].

\section{Körperliche Fitness}

Im Rahmen der Erhebung der körperlichen Fitness der Probanden wurden folgende Testungen durchgeführt: Handgriffkraft, Medizinballweitwurf, Standweitsprung, Sit and Reach, Einbeinstand, $10 \mathrm{~m}$ Sprint und $20 \mathrm{~m}$ Sprint, Andersen-Test und Messung der körperlichen Aktivität.

\section{Ergebnisse}

\section{Körperfett}

Die Probanden der Kontrollgruppe wiesen vor Interventionsbeginn einen signifikant niedrigeren Körperfettanteil auf als alle Schüler, die der Interventionsgruppe zugeordnet waren $(p=0,025)$. Bei der ersten Follow-up-Messung nach drei Monaten und Partizipation des ersten Interventionszyklus konnte kein signifikanter Unterschied zwischen Kontrollund Interventionsgruppe identifiziert werden. Ebenso unterschieden sich die beiden Gruppen nach Partizipation des zweiten Interventionszyklus hinsichtlich ihres Körperfettanteils nicht signifikant, wobei jedoch darauf zu verweisen ist, dass die Probanden der Interventionsgruppe zu diesem Zeitpunkt einen nicht signifikant niedrigeren $(p=0,075)$ Körperfettanteil aufwiesen als die der Kontrollgruppe zugeordneten Teilnehmer.

\section{Blutparameter}

Vor Interventionsbeginn wurden 19 Probanden $(n=139)$ mit erhöhten LDLWerten identifiziert, erhöhte Lipoprotein-(a)-Werte wurden bei 34 und erhöhte Triglyceride bei 41 Probanden gemessen.

Auffallend war eine hohe Prävalenz von Vitamin-D-Unterversorgungen (120 Probanden, $n=138$ ) innerhalb der Stichprobe, wobei bei 19,6\% Probanden ein massives Defizit festgestellt wurde. Darüber hinaus lag bei einem auffallend hohen Anteil von rund $18 \%$ der Probanden ein Vitamin-B B $_{12}$-Mangel vor. Rund $42 \%$ der Probanden hatten verringerte Ferritinwerte. Bei $7 \%$ der Schüler zeigte sich ein manifester Eisenmangel (• Tab. 2).

\section{Ernährungswissen}

Vor Intervention beantworteten die Probanden der Interventionsgruppe durchschnittlich 49,7\% der zwölf gestellten Fragen korrekt. Der Vergleich des Wissensstandes vor und nach Intervention zeigt einen Wissensanstieg von durchschnittlich $7,4 \%$ in der Interventionsgruppe direkt nach Intervention im Vergleich zum Basiszeitpunkt und eine weitere Erhöhung um 2,8\% im Follow-up nach fünf Monaten. Insgesamt hat sich das Ernährungswissen nach Intervention im Vergleich zur Basiserhebung vor Interventionsbeginn um durchschnittlich $8,1 \%$ verbessert, was einer signifikanten Erhöhung des Ernährungswissens entspricht $(p=0,000)$ (• Abb. 1).

\section{Ernährungsverhalten}

Der Vergleich ausgewählter Lebensmittel zeigt eine signifikante Reduktion des Konsums von folgenden Lebensmittelgruppen in der Interventionsgruppe vor und nach Intervention: 
- Fast-Food-Produkte $(p=0,05)$,

- Süßigkeiten $(p=0,01)$,

- salzige Snacks $(p=0,01)$.

\section{Psychische Konstitution} und Einflussfaktoren auf die Entstehung von Übergewicht und Adipositas

Vor Beginn der Intervention gaben $73 \%$ der Probanden an, sich selbst als schlank oder normalgewichtig zu sehen. Laut BMI fallen nur rund $63 \%$ der Probanden der Stichprobe in den Normalbereich. Somit wiesen $10 \%$ der Schüler vor Beginn der Intervention eine verzerrte Selbstwahrnehmung hinsichtlich ihrer eigenen Körpermaße auf und unter- oder überschätzen ihre eigene Körperkomposition.

Erhebungen zur Bewegungsmotivation (• Abb. 2) ergaben, dass rund $28 \%$ der Schüler keine Freude an Bewegung empfanden und diese nicht als herausfordernd bzw. aufregend wahrnahmen.

Mädchen befassten sich signifikant häufiger mit dem Thema Figur und Gewicht als Buben $(p=0,05)$, während das physische Wohlbefinden bei den Buben signifikant höher war als das selbst reportierte körperliche Wohlbefinden der Mädchen $(p=0,031)$.

\section{Diskussion}

Bisher publizierte kontrollierte Studien beschreiben die Effekte von Präventionsmaßnahmen auf Übergewicht und kardiovaskuläre Risikofaktoren bei Kindern und Jugendlichen. Als Haupteinflussfaktoren wurden dabei Ernährungs- und Bewegungsinterventionen identifiziert, welche durch eine Verbesserung des Gesundheitswissens das Gesundheitsverhalten von Kindern und Jugendlichen nachhaltig positiv beeinflussen und somit das Risiko für die Entwicklung von Übergewicht und dessen Folgeerkrankungen reduzieren [7-9].

Gemäß des multifaktoriellen Ansatzes zur Verbesserung der positiven Gesundheit wird neben körperlichen und sozialen Indikationsfeldern ebenso die Berücksichtigung psychologischer Faktoren betont [10]. Demnach stellen psychologische Aspekte eine zentrale Di-

Paediatr Paedolog 2016 · 51:104-108 DOI 10.1007/s00608-016-0371-y

(c) The Author(s) 2016 . This article is available at SpringerLink with Open Access

\section{Pöppelmeyer $\cdot$ O. Helk $\cdot$ S. Mehany $\cdot$ R. Hauer $\cdot$ O. Pachinger $\cdot$ K. Widhalm Die Wiener Präventionsstudie EDDY - Erste Ergebnisse}

\section{Zusammenfassung}

Übergewicht und Adipositas sind ein zentrales Gesundheitsproblem in Europa. Die Weltgesundheitsorganisation (WHO) erklärte die Prävention von Übergewicht zu einem wissenschaftlichen Forschungsziel mit oberster Priorität.

Mit dem Ziel den Gesundheits- und Ernährungsstatus eines repräsentativen Samples von Wiener Schülerinnen und Schülern im Alter von 11 bis 14 Jahren zu erheben und die Auswirkungen einer Intervention mit Ernährungs- und Bewegungsschulungen auf die Körperzusammensetzung, metabolische Faktoren und das Ernährungswissen und -verhalten zu erfassen, wurde im Rahmen des Präventionsprojektes „EDDY“ eine zweijährige Interventionsstudie an vier Wiener Schulen durchgeführt.

Die Ergebnisse verdeutlichen, dass das Ernährungswissen und in einigen Bereichen auch das Ernährungsverhalten durch standardisierte Ernährungs- und Lebensstilintervention verbessert werden können.

Hinsichtlich des körperlichen Gesundheitszustandes zeigt sich, dass der Körperfettanteil in der Interventionsgruppe nach Intervention niedriger war als in der Kontrollgruppe. Bemerkenswert sind zudem eine außerordentlich große Anzahl an Probanden mit unzureichender Vitamin-D-Versorgung $(86,95 \%)$ sowie ein erheblicher Anteil an Schülern mit Vitamin-B ${ }_{12}$-Unterversorgung $(17,98 \%)$.

Bedauerlich ist, dass $28 \%$ der Schüler angaben, keine Freude an körperlicher Aktivität zu empfinden.

Hinsichtlich der Infrastruktur in den Schulen muss auf eine zum Teil sehr mangelhafte Qualität und Größe der Turnsäle hingewiesen werden. Darüber hinaus war die ausreichende professionelle Betreuung durch sportwissenschaftlich ausgebildetes Fachpersonal in einigen Schulen nicht gegeben. Auch eine Involvierung der Schulärzte in das Präventionsprojekt war kaum möglich.

Schlüsselwörter

Prävention · Public Health - Übergewicht . Ernährungsintervention

\section{The Viennese Prevention Study (EDDY): initial results}

\section{Abstract}

Excess weight and obesity are considerable health problems in Europe. The World Health Organization (WHO) declared the prevention of excess weight to be a scientific research objective of the highest priority.

The prevention project "EDDY", an interventional study carried out in four Viennese schools and lasting 2 years, was conducted with the aim of detecting the health and nutritional status of a representative sample of Viennese school pupils aged 11 to 14 years and to measure the effects of an intervention with diet and exercise training on body composition, metabolic factors, and nutritional knowledge and behavior.

The results show that nutritional knowledge and in some areas eating habits can be improved through standardized diet and lifestyle intervention.

Regarding physical health status, a lower body fat percentage in the intervention group was shown after the intervention compared with the control group.

It is noteworthy that there were an extraordinarily large percentage of subjects with insufficient vitamin D supply ( $86.95 \%$ ) and a considerable proportion of pupils with vitamin B12 deficiency (17.98\%). Regrettably, $28 \%$ of students reported that they feel no pleasure in physical activity. Regarding the infrastructure in the schools, the sometimes highly deficient quality and size of the gyms should be pointed out. Furthermore, there was inadequate professional care by scientifically trained sports specialists in some schools. Also, the involvement of school doctors in the prevention project was hardly ever possible.

\section{Keywords}

Prevention - Public health - Excess weight . Nutritional intervention 


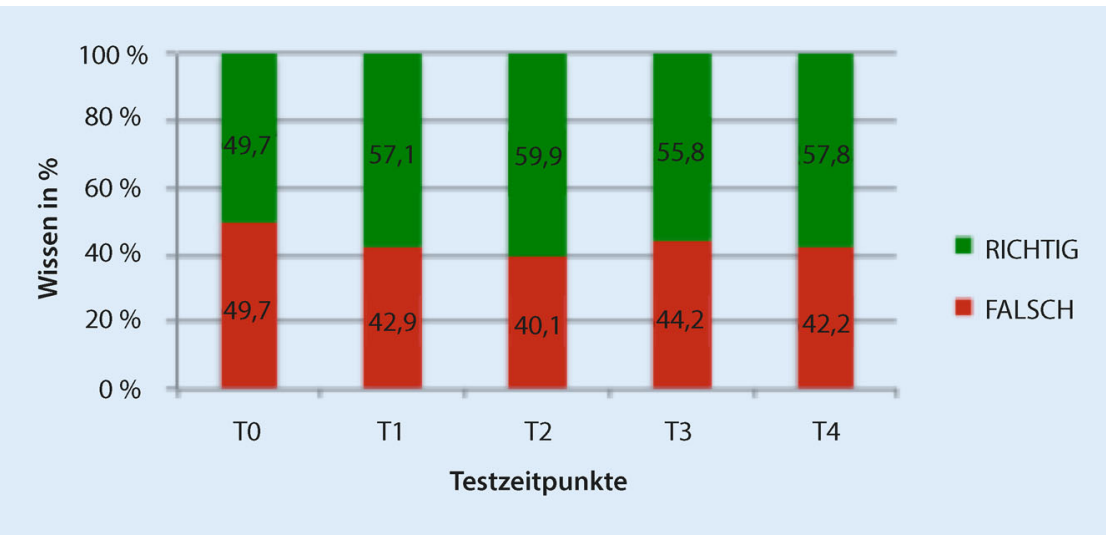

Abb. $1 \Delta$ Ernährungswissen der Probanden zu verschiedenen Testzeitpunkten $\odot$ ÖAIE

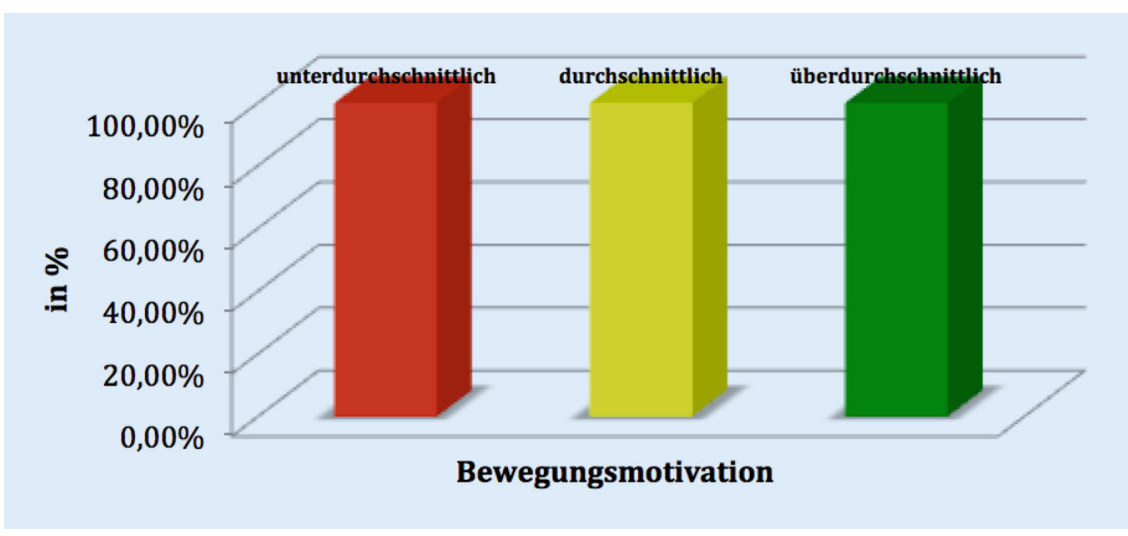

Abb. $2 \triangle$ Bewegungsmotivation der Probanden ๑ ÖAIE

mension in der Präventionsarbeit dar. Die psychologischen Daten der EDDYStudie verdeutlichen die Wichtigkeit dieser Dimension jedoch weiter und weisen darauf hin, diese dritte Dimension positiver Gesundheitsentwicklung künftig noch stärker zu berücksichtigen. Die vorliegenden Ergebnisse weisen dabei besonders auf eine verzerrte Wahrnehmung des eigenen Körperschemas der Schülerinnen und Schüler hin. Vor allem Mädchen zeigen dabei im Vergleich zu ihren männlichen Mitschülern ein deutlich vermindertes körperliches Wohlbefinden. Insgesamt ist die Auseinandersetzung mit dem Thema Figur und Gewicht für Schülerinnen der untersuchten Altersgruppe deutlich intensiver. Gerade diese könnten daher besonders von psychologischen Interventionen profitieren.

Eine Hauptzielgröße von Gesundheitspräventionsmaßnahmen ist die Verbesserung des Wissens im forcierten Interventionsbereich durch spezifische Schulungen. Zur Beurteilung der Ent- kanten Ausmaß im Vergleich zum Zeitpunkt vor Interventionsbeginn. Erwähnenswert ist dabei, dass der mittlere KFA in der Interventionsgruppe zum Basiszeitpunkt höher war als der durchschnittliche KFA der Probanden der Kontrollgruppe, während die Probanden der Interventionsgruppe bei Vergleich post interventionem einen geringeren KFA aufwiesen als jene Probanden, welche an der Intervention nicht partizipierten.

Neben dem Edukationserfolg und Gesundheitszustand ist auch das Gesundheitsverhalten eine wichtige Zielgröße von Präventionsmaßnahmen. Der Prä-/ Post-Vergleich des Konsums von Fast Food, Süßigkeiten und salzigen Snacks der Probanden der Interventionsgruppe weist eine signifikante Reduktion des Konsums aller drei Lebensmittelgruppen nach Absolvierung des Schulungsprogrammes auf.

Darüber hinaus konnten im Rahmen der Interventionsstudie einige Probanden mit pathologischen Blutwerten identifiziert werden. So ergab die Bestimmung der Blutparameter zum Basiszeitpunkt, dass bei rund $87 \%$ aller Teilnehmer ein Vitamin-D-Defizit vorlag. Mehr als ein Viertel der Schüler wiesen erhöhte Triglycerid- und Lipoprotein-(a)-Werte auf und mehr als ein Drittel der Probanden hatte verminderte Ferritinkonzentrationen im Blut.

Insgesamt lassen die Ergebnisse darauf schließen, dass eine Verbesserung des Ernährungswissens durch ein kombiniertes Ernährungs- und Bewegungstraining im Rahmen einer schulbasierten Präventionsmaßnahme sowohl das Gesundheitsverhalten als auch den Gesundheitszustand der Probanden positiv beeinflusst. Diese Ergebnisse reihen sich in den Wissensstand weniger aktueller kontrollierter Präventionsstudien ein, welche einen Benefit von Ernährungsund Bewegungsschulungen auf den Gesundheitszustand von Schülern beschreiben, und zeigen Evidenz dafür, dass eine altersgerechte Ernährungs- und Bewegungsintervention positive Änderungen im Lebensstil der Schüler bewirken kann $[6,8,9]$. 


\section{Fazit für die Praxis}

- Mit der vorliegenden Studie konnte gezeigt werden, dass bei 11- bis 14jährigen Schülern durch standardisierte Ernährungs- und Lebensstilintervention eine Verbesserung des Ernährungswissens und in einigen Bereichen des Ernährungsverhaltens sowie des körperlichen Gesundheitszustandes erzielt werden kann.

- Bemerkenswert ist eine außerordentlich große Anzahl an Probanden mit unzureichender Vitamin-D-Versorgung $(86,95 \%)$ sowie ein erheblicher Anteil an Schülern mit Vitamin-B ${ }_{12}$ Unterversorgung (17,98\%).

- Hinsichtlich der Infrastruktur in den Schulen muss auf eine zum Teil sehr mangelhafte Qualität und Größe der Turnsäle hingewiesen werden. Darüber hinaus war die ausreichende professionelle Betreuung durch sportwissenschaftlich ausgebildetes Fachpersonal in einigen Schulen nicht gegeben. Auch war eine Involvierung der Schulärzte in das Präventionsprojekt kaum möglich.

\section{Korrespondenzadresse}

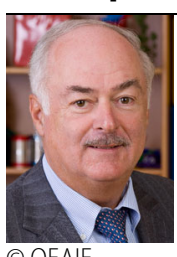

Prof. Dr. K. Widhalm

Österreichisches Akademisches Institut für Ernährungsmedizin Alserstraße 14/4a, 1090 Wien Österreich kurt.widhalm@ meduniwien.ac.at

\section{Einhaltung ethischer Richtlinien}

Interessenkonflikt. C. Pöppelmeyer,O. Helk, S. Mehany, R. Hauer und O. Pachinger, K. Widhalm geben an, dass kein Interessenkonflikt besteht.

Alle beschriebenen Untersuchungen wurden mit Zustimmung der zuständigen Ethik-Kommission, im Einklang mit nationalem Recht sowie gemäß der Deklaration von Helsinki von 1975 (in der aktuellen, überarbeiteten Fassung) durchgeführt. Von allen beteiligten Patienten liegt eine Einverständniserklärung vor.

Open Access. This article is distributed under the terms of the Creative Commons Attribution 4.0 International License (http://creativecommons.org/ licenses/by/4.0/), which permits unrestricted use, distribution, and reproduction in any medium, provided you give appropriate credit to the original author(s) and the source, provide a link to the Creative Commons license, and indicate if changes were made.

\section{Literatur}

1. World Health Organization (2000) Obesity: Preventing and managing a global epidemic Report of WHO consultation. WHO Technical Report Series, Bd.no. 894. WHO, Geneva

2. Diethelm K, Huybrechts I, Moreno L, De Henauw $S$, Manios Y, Beghin L, González-Gross M, Le Donne C, Cuenca-García M, Castillo MJ, WidhalmK, Patterson E, Kersting M (2014) Nutrient intake of European adolescents: results of the HELENA (Healthy Lifestyle in Europe by Nutrition in Adolescence) Study. Public Health Nutrition 17:486-497

3. Statistik Austria (2010) Todesursachenkategorien. http://www.statistik.at/web_de/statistiken/ gesundheit/todesursachen/todesursachen ausgewaehlte/index.html. Zugegriffen: 5. Jan. 2015

4. Grundy SM (2012) Pre-diabetes, metabolic syndrome, and cardiovascular risk. J Am Coll Cardiol 59:635-643

5. Kromeyer-Hauschild K, Wabitsch M, Kunze D, Geller F, Geiß HC, Hesse V, von Hippel A, Jaeger U, Johnsen D, Korte W, Menner K, Müller G, Müller JM, Niemann-Pilatus A, Remer T, Schaefer F, Wittchen HU, Zabransky S, Zellner K, Ziegler A, Hebebrand $\mathrm{J}$ (2001) Perzentile für den Body-mass-Index für das Kindes- und Jugendalter unter Heranziehung verschiedener deutscher Stichproben. Monatsschr Kinderheilkd 149:807-818

6. Ardelt-Gattringer E, Meindl M (2014) Interdisziplinäres Testsystem zur Adipositas- Diagnostik und Evaluation bei Adipositas und anderen durch Ess- und Bewegungsverhalten beeinflussbare Erkrankungen (Modul 1). Wirtz MA (Hrsg) Dorsch - Lexikon der Psychologie. 17. Aufl. Bern: Verlag Hans Huber, S769

7. Jouret B, Ahluwalia N, Dupuy M, Cristini C, NègrePages L, Grandjean H, Tauber M (2009) Prevention of overweight in preschool children: results of kindergarten-based interventions. Int J Obes 33:1075

8. Nemet D, Barkan S, Epstein Y, Friedland O, Kowen G, Eliakim A (2005) Short- and long-term beneficial effects of a combined dietary-behavioral-physical activityintervention for the treatment of childhood obesity. Pediatrics 115:443-449

9. Yin Z, Moore JB, Johnson MH, Vernon MM, Gutin B, Zenong $Y$ (2012) The impact of a 3-year afterschool obesity program in elementary school children. Child Obes 8:60-70

10. Pelikan JM (2009) Ausdifferenzierung von spezifischen Funktionssystemenfür Krankenbehandlung und Gesundheitsförderung oder: Leben wir in der "Gesundheitsgesellschaft"? Österreichische Z Soziol 34:28-47

11. Expert Panel on integrated Guidelines for cardiovascular health and risk reduction (2011) Expert Panel on Integrated Guidelines for Cardiovascular health and risk reduction in children and adolescents: summary report. Pediatrics 128:213-256

12. González-Gross M, Benser J, Breidenassel C, Albers U, Huybrechts I, Valtueña J, Spinneker A, Segoviano M, Widhalm K, Molnar D, Moreno LA, Stehle P, Pietrzik K (2012) Gender and age influence blood folate, vitamin B12, vitamin B6, and homocysteine levels in European adolescents: the HelenaStudy. Nutrition Research 32:817-826
13. González-Gross M, Valtueña J, Breidenassel C, Moreno LA, Ferrari $M$, Kersting $M$, De Henauw S, Gottrand F, Azzini E, Widhalm K, Kafatos A Manios Y, Stehle P (2012) Vitamin D status among adolescents in Europe: the Healthy Lifestyle in Europe by Nutrition in Adolescence study. British Journal of Nutrition 107:755-764 